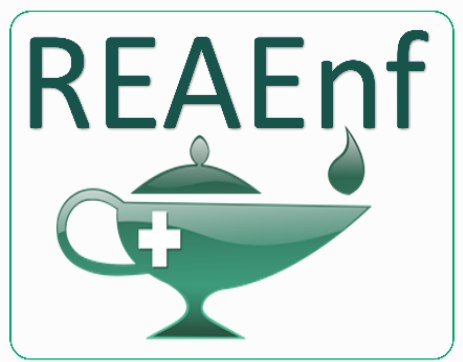

Revista Eletrônica Acervo Enfermagem
REVISÃO BIBLIOGRÁFICA

Recebido em: 10/2020

Aceito em: $11 / 2020$

Publicado em: 12/2020

\title{
A força da resiliência: um estudo de revisão sobre as contribuições do pensamento lateral no trabalho do enfermeiro
}

\author{
The strength of resilience: a review study on the contributions of side thought in nurses \\ 'work
}

La fuerza de la resiliencia: un estudio de revisión de las contribuciones del pensamiento lateral en el trabajo de las enfermeiras

Faraildes Maria Cavalcanti Almeida1, Iris Edná Pereira da Silva ${ }^{2 \star}$, Érica Edileuza Santos Leal Ferreira $^{1}$, Virgínia lone Araújo de Souza ${ }^{3}$, Dálete de Souza Coelho Silva ${ }^{3}$.

\begin{abstract}
Resumo: A resiliência consiste na capacidade de o indivíduo se manter bem, recuperar-se, e ser bemsucedido frente às adversidades, que reflete na capacidade de ultrapassar limites interiores em situações de riscos do contexto psicossocial. Este artigo consiste em um estudo exploratório com abordagem qualitativa, na modalidade de revisão de literatura. É discutido o conceito de resiliência e pensamento lateral, os quais contribuem para potencializar as ações dos enfermeiros, tornando-os motivados, focados, positivos, flexíveis, organizados e pró-ativos. A conclusão da revisão demonstra, no entanto, que a literatura pertinente à resiliência dos enfermeiros revela pontos reflexivos. Contudo, com a aplicação dos conceitos do pensamento lateral e da resiliência na execução das atividades dos enfermeiros a estimulação da imaginação criativa possibilitam a ultrapassar diversas barreiras. Apesar da sobrecarga de atividades os enfermeiros conseguem utilizar seus talentos criativos e encontram novas soluções para os problemas, para contribuir com o crescimento e o desenvolvimento das instituições de saúde.
\end{abstract}

Palavras-chave: Resiliência psicológica, Enfermagem, Pensamento lateral.

Abstract: Resilience consists of the individual's ability to remain well, recover, and be successful in the face of adversity, which reflects in the ability to overcome inner limits in situations of risk in the psychosocial context. This article consists of an exploratory study with a qualitative approach, in the form of literature review. The concept of resilience and lateral thinking is discussed, which contribute to enhance nurses' actions, making them motivated, focused, positive, flexible, organized and proactive. The conclusion of the review demonstrates, however, that the literature relevant to nurses' resilience reveals reflexive points. However, with the application of the concepts of lateral thinking and resilience in the performance of nurses' activities, the stimulation of creative imagination makes it possible to overcome various barriers. Despite the overload of activities, nurses are able to use their creative talents and find new solutions to problems, to contribute to the growth and development of health institutions.

Keywords: Resilience, Nursing, Lateral thinking.

\footnotetext{
1 Faculdade Maurício de Nassau, Recife - PE.

2 Faculdade de Ciências Médicas (UPE), Recife - PE.*E-mail: iris.psilva@upe.br

3Universidade de Pernambuco, Recife - PE.
} 
Resumen: La resiliencia consiste en la capacidad del individuo para mantenerse bien, recuperarse y tener éxito frente a la adversidad, lo que se refleja en la capacidad de superar los límites internos en situaciones de riesgo en el contexto psicosocial. Este artículo consiste en un estudio exploratorio con enfoque cualitativo, en forma de revisión de la literatura. Se discute el concepto de resiliencia y pensamiento lateral, que contribuyen a potenciar las acciones de las enfermeras, haciéndolas motivadas, enfocadas, positivas, flexibles, organizadas y proactivas. La conclusión de la revisión demuestra, sin embargo, que la literatura relevante a la resiliencia de las enfermeras revela puntos reflexivos. Sin embargo, con la aplicación de los conceptos de pensamiento lateral y resiliencia en el desempeño de las actividades del enfermero, la estimulación de la imaginación creativa permite superar diversas barreras. Apesar de la sobrecarga de actividades, las enfermeras pueden utilizar su talento creativo y encontrar nuevas soluciones a los problemas, para contribuir al crecimiento y desarrollo de las instituciones de salud.

Palabras clave: Resiliencia, Enfermería, Pensamiento lateral.

\section{INTRODUÇÃO}

A capacidade de superar as mais difíceis tragédias e de se tornarem mais fortes ganhou recentemente a denominação de resiliência. Resiliência é um conceito emprestado da física, que tem a ver com a propriedade de que são dotados alguns materiais, de acumular energia quando exigidos ou submetidos a estresse, voltando em seguida ao seu estado original, sem qualquer deformação. O comportamento resiliente se opõe àquele em que certas pessoas, submetidas a algum trauma, tornam-se incapazes de reação (GOLDSTEIN TS, 2012).

Neste sentido, os estudos e as pesquisas realizados sobre resiliência humana procuram compreender porque, diante das mesmas condições adversas, alguns indivíduos conseguem desenvolver-se, ultrapassando os limites da condição humana, enquanto outros são vencidos, perdem o ânimo e desenvolvem patologias ou se menosprezam (BIANCHINI DSC e DELL'AGLIO DD, 2007).

A resiliência consiste na capacidade de o indivíduo se manter bem, recuperar-se, e ser bem-sucedido frente às adversidades. Esta incrível força de se levantar e arranjar entusiasmo funciona como um conjunto de forças psicológicas e biológicas que são fundamentais para se atravessar com sucesso às mudanças. Para tanto, é necessário que os processos fisiológicos, que são ativados pelo estresse, o tornem resiliente através da habilidade de conhecer a dor, e de aceitá-la até decidir os conflitos de maneira construtiva (BRANDÃO JM, et al., 2011).

O estudo do tema considera aqueles indivíduos que, apesar das predições contrárias atinentes às situações de riscos do contexto psicossocial em que estão inseridos, tiveram êxito diante da adversidade ou cresceram e se desenvolveram a partir dela (MENDES JM, 2018).

Nesta mesma linha, Brant LC e Dias EC (2004) considera que, para o desenvolvimento da resiliência, as pessoas devem possuir atributos básicos, tais como: pessoas positivas, focadas, flexíveis, organizadas e proativas, e, de acordo com Bianchini DSC e Dell'Aglio DD (2007), com autoestima positiva, habilidade de dar e receber nas relações com o outro, disciplina pessoal e sentido de responsabilidade, reconhecimento e desenvolvimento de seus próprios talentos; mente aberta e receptiva a novas ideias; interesse; tolerância ao sofrimento; esperança; criatividade; e autonomia.

A sociedade atual requer mudanças constantemente, levando o indivíduo a ser cada vez mais competitivo, e para que o mesmo possa sobreviver e crescer nela, é preciso reagir aos impactos ocasionados por essas transformações, visando, com isso, obter uma melhor condição de vida que será traduzida pelo seu bemestar. As pessoas mais resilientes estão mais aptas para enfrentar situações difíceis, absorvendo mais rapidamente as dificuldades que surgem, e continuam desenvolvendo suas habilidades até atingir seus objetivos. Por possuir mais de um emprego e conseguir cumprir os horários e as atividades relativas aos mesmos, torna-se, dessa forma, objeto de estudo saber: De onde vem a resistência física e mental do enfermeiro para atuar em mais de um emprego (BIANCHINI DSC e DELL'AGLIO DD, 2007). 
Desta forma, o presente artigo objetiva analisar as contribuições do pensamento lateral no trabalho desenvolvido pelo enfermeiro resiliente. Especificamente, pretende-se: Identificar as características de resiliência; identificar no enfermeiro resiliente se a sobrecarga de trabalho reflete na sua saúde física e mental; identificar novos paradigmas da qualidade do trabalho do enfermeiro resiliente.

\section{REVISÃO BIBLIOGRÁFICA}

\section{Resiliência e sintomas físicos do estresse nos enfermeiros}

Sendo a alienação um distúrbio das paixões, Peixoto ALS (2013) acreditava ser possível tratá-la reeducando a mente alienada, considerando a doença como uma reação salutar do organismo contra a ação de causas que perturbavam seu equilíbrio. Em um estudo realizado por Macedo ABT et al. (2020) mostrou que $(74,36 \%)$ dos profissionais perceberam alto esforço na realização do trabalho e o estresse psicossocial foi identificado em $(69,23 \%)$. Foi verificado que $(56,41 \%)$ apresenta baixa resiliência concomitantemente com baixa recompensa por parte da instituição em promover o incentivo para melhorias ao processo de trabalho. Geralmente, o estresse e a má adaptação são precursores da doença, quando este estado é prolongado ou a resposta é excessiva, ele aumentará a susceptibilidade da pessoa à doença (MACEDO ABT, et al., 2020).

O surgimento da doença mental está associado à dificuldade de adaptação do indivíduo, diante de situações de sofrimento. Dentre os transtornos mentais que podem ser desencadeados em tal situação, destacam-se os Transtornos Mentais Comuns (TMC), caracterizados por situações de sofrimento psíquico, que sinalizam uma anormalidade no funcionamento psíquico, apresentando sintomas como: insônia, fadiga, irritabilidade, esquecimento, dificuldades de concentração e queixas somáticas (CECHIN P, et al., 2014).

Os TMC ocorrem quando há alterações orgânicas significativas mediante a presença do estímulo estressor, segundo Souza MTS e Cerveny CMO (2005) é a partir desse ponto que começam as reações orgânicas, as atitudes, as emoções e comportamentos, alterações químicas, fisiológicas, dentre outras. Psiquicamente, a ansiedade crônica ou esgotamento leva a um estado de apatia e desinteresse, desânimo, uma espécie de pessimismo, insegurança e medo em relação à vida (SOUZA MTS e CERVENY CMO, 2005).

Outro aspecto relevante refere-se ao estado emocional dos enfermeiros, pois o estresse psicossocial está relacionado ao grau de exigência do trabalho, bem como aos recursos disponíveis para desenvolvê-lo, gerando sofrimento psíquico, devido a incapacidade para realizar as tarefas laborais. As ansiedades geradas pelo stress pode também ser positivas, mesmo tendo influência de aspectos negativos na profissão como: processos de trabalhos burocráticos, cultura de punição, sobrecarga e dupla jornada de trabalho, uma vez que o estresse impulsiona os enfermeiros a buscar estratégias para ultrapassar as dificuldades no seu cotidiano através da resiliência (MACEDO ABT, et al., 2020).

Como se pode perceber, o adoecimento mental tem causa multifatorial e o indivíduo que desenvolve a doença mental, muitas vezes se sente em sofrimento, desesperançado e incapaz de levar a vida na sua plenitude. Há uma preocupação constante em termos de saúde pública, pois os transtornos mentais podem causar incapacidade para o trabalho, retraimento social e maior predisposição a desenvolver doenças físicas (MACEDO ABT, et al., 2020).

Vários estudos abordam a associação de sofrimento psíquico e o trabalho; dentre eles, um realizado em gestores verificou que qualquer que seja a direção, o trabalho parece constituir-se numa fonte de sofrimento (BRANT LC e DIAS EC, 2004). Ou seja, de acordo com o autor, tanto o trabalho que apresenta níveis de desgaste físico, quanto o trabalho que necessita de capacidades gerenciais, como liderança e tomada de decisões, podem gerar o sofrimento psíquico. Entende-se, por outro lado, que a dupla jornada de trabalho se faz necessária aos trabalhadores de enfermagem, devido à situação econômica da área da saúde, aos baixos salários insuficientes para o sustento da família, o que os leva a procurar novas fontes de renda. Na realidade, necessitam enfrentar dupla atividade propiciando o estresse, podendo também afetar o desempenho físico, interferindo em alguns aspectos referentes à qualidade de vida do trabalhador, ou seja, os profissionais muitas vezes reconhecem as alterações ocasionadas no serviço e que estão expostos a desenvolver, mas, porém, preferem manter essa rotina em busca de melhorias no futuro (CECHIN P, et al., 2014). 
Atualmente, o estresse significa pressão, insistência e estar estressado significa estar sob pressão ou estar sob ação de um determinado estímulo insistente. Tem sido considerado como um dos problemas que mais frequentemente age sobre o ser humano, interferindo na homeostase de seu organismo, devido à grande quantidade de tensões que enfrenta diariamente (SILVA JLM e MELO ECP, 2006).

A vulnerabilidade dos indivíduos ao estresse depende da sua habilidade para lidar com os eventos estressores. Não só estes, mas a maneira como o indivíduo lida com eles é fundamental para que se desenvolva um quadro de estresse. Uma vez que o modo de reagir a estímulos é um produto da aprendizagem, o contrário também pode ocorrer e é possível desaprender certas reações inadequadas e estressoras em potencial (SÓREA DAC, et al., 2006).

No estudo feito sobre a capacidade de resiliência, foi verificado que algumas pessoas, apesar das grandes mudanças, apresentavam um pequeno grau de disfunção. As disfunções podem ser observadas através de diversos comportamentos, entre os quais podemos citar: decisões mal tomadas, constantes conflitos com parceiros de trabalho, irritação momentânea que pode interromper a atenção na execução de suas tarefas, comportamento explosivo inadequado no ambiente empresarial, frustrações do trabalho afetando o relacionamento familiar, entre tantos outros. Em um estágio mais avançado, tais disfunções podem causar: moral baixa, dores de cabeça e/ou de estômago, apatia, depressão crônica, esgotamento físico e/ou psicológico, levando, até mesmo, ao suicídio (SILVA JLM e MELO ECP, 2006).

\section{Resiliência e promoção da saúde}

Compreende-se que a política da promoção da saúde surge para fortalecer e implantar a transversalidade do cuidado integral e intersetorial, favorecendo o diálogo entre as todas as áreas do setor saúde, quer sejam públicas, filantrópicas, Organizações Sociais (Os) e o setor privado complementar ao Sistema Único de Saúde (SUS), formando as redes de acordos e compromissos mútuos, as quais visam melhorar a qualidade dos serviços prestados pelo SUS, atendendo a população em todas as suas necessidades, objetivando romper com a fragmentação do cuidado, diminuindo os danos, os riscos, as vulnerabilidades e as iniquidades em saúde (BRASIL, 2010).

Ressalta-se que a resiliência é a capacidade das pessoas se sobressair e se reinventar diante de situações adversas e os enfermeiros que estão inseridos no processo do cuidar tornam-se resilientes, pois passam por várias situações estressantes e barreiras, que são impostas no seu cotidiano profissional, especialmente no que se refere a sobrecarga de trabalho, pois, como não são bem remunerados, necessitam trabalhar em dois ou três empregos, a fim de ter condições de proporcionar qualidade de vida a si e aos seus familiares.

Entretanto, eles só conseguem ultrapassar as dificuldades nas suas atividades laborais buscando coragem e pensando positivamente, focando no cuidado ao paciente, na melhor maneira de inseri-lo no seu processo de cura, não deixando que a doença seja o centro do cuidado como acontecia no modelo médico assistencial privatista, e sim admitir que seja ampliado o processo saúde doença, proporcionado um cuidado integral do cliente, onde sua família, sua comunidade e a equipe de enfermagem participem ativamente desse cuidado (SILVA MJSM, et al., 2019; MOTA BS, et al., 2020) .

Outras importantes estratégias de resiliência estão descritas na literatura como a religiosidade e espiritualidade no cotidiano do idoso que refletem na qualidade de vida e aliviam os sintomas depressivos, a esperança como um aspecto resiliente em pessoas em tratamento hemodialítico, crianças em estágios terminais na terapia intensiva entre outros, são pontos cruciais para manutenção dos conceitos de promoção da saúde, garantindo de forma humanizada esses preceitos. A correlação positiva com a resiliência coloca a empatia como essencial atributo ao cuidado prestado, sendo o enfermeiro o agente impulsionante desse processo (REIS LA e MENEZES TMO, 2017; FERREIRA C, et al., 2018; LIMA GS, et al., 2019; ANDRADE BRV, et al., 2020).

Nesta perspectiva, os estudos sobre a promoção da saúde dos enfermeiros tem alertado diversas instituições de saúde para promover mudanças na gestão do trabalho, isto é, nas práticas institucionais tornado os ambientes de trabalho saudáveis, realizando a prevenção das doenças relacionadas ao trabalho, como a Lesão por Esforço Repetitivo (LER), através de inserção do profissional de educação física realizando 
ginástica laboral, bem como realizar capacitação dos colaborados, a fim de mantê-los atualizados, e dirimir dúvidas referentes aos atuais protocolos assistenciais (CRUZ EJER, et al., 2018)

Neste sentido, os estudos sobre a promoção da saúde dos enfermeiros revelam que os profissionais que possuem dupla carga horária de trabalho encontram forças para conseguir realizar suas atividades sendo: positivos, tendo lazer, bom humor, possuindo uma rede de relacionamento interpessoal e recebendo o apoio da família, como pontos importantes para manutenção do trabalho (CECHIN P, et al., 2014).

Assim, nota-se que é necessário que os empregadores promovam estratégias que se articulem por meio de ações interdisciplinares e intersetoriais para colaborar no enfrentamento dos problemas ocasionados pelo excesso de trabalho, como a LER, o estresse emocional e o desgaste físico.

Com isto, as evidências destacam a percepção dos líderes e colaboradores em ofertar atividades para estímulos alternativos e dinâmicos que se enquadrem no âmbito do cuidado integral com a saúde do trabalhador, ações que visem promoção da saúde em ambientes saudáveis e prevenção de agravos ocupacionais que reflitam na implementação de pausas para exercícios laborais e para descanso (CRUZ EJER, et al., 2018).

\section{Resiliência e o pensamento lateral como instrumentos facilitadores na quebra de paradigmas pelo enfermeiro}

O pensamento lateral foi estudado por Edward de Bono no período de 1960 a 1970, através de suas pesquisas sobre o pensamento humano as formas de aumentar o pensamento criativo, avaliando 0 comportamento do cérebro. Neste sentido, o pensamento lateral baseia-se na quebra de percepções desenvolvido pelo modelo auto-organizável do cérebro, uma vez que ele constitui uma sequência de atividades com as primeiras informações recebidas, as quais com o tempo seguem um caminho favorito e com isso, percebeu-se que o cérebro passa a reconhecer padrões de pensamento, os quais passam a serem utilizados em várias formas de relacionamento (BONO E, 2003; SÓREA DAC, et al., 2006).

Neste contexto, o pensamento lateral é um pensamento diferente, pois ele leva a pessoa a criar novas soluções para os problemas, através da estimulação do cérebro a criar por caminhos laterais, isto é, estimulando o cérebro a ter novas formas de se desenvolver, levando-o a fluir por áreas que não eram usadas anteriormente. Com isso, percebeu-se que devemos considerar também as ideias com poucas probabilidades de serem aceitas no curso normal dos acontecimentos, porque elas serão fundamentais para criar soluções novas para os problemas (SÓREA DAC, et al., 2006).

As organizações de saúde, precisam de pessoas criativas, que pensem de modo não-convencional, que sejam levadas a desenvolver todo o seu potencial para propor novas estratégias, novos serviços, e novas maneiras para realizar atividades, deixando aflorar seu poder criativo, a fim de atender as necessidades das instituições de saúde, tornando-as competitivas, modernas e acessíveis à população (CECHIN P, et al., 2014).

Nesse sentido, o pensamento lateral contribui para o desenvolvimento da criatividade, na medida em que colabora no processo da criação de soluções, as quais são afloradas pela resiliência do enfermeiro, que ultrapassa paradigmas antigos para renovar-se e contribuir com inovações nos processos de trabalho, tornando rotinas mais práticas e de fácil execução pela equipe de enfermagem, possibilitando ao enfermeiro uma liderança democrática, onde os subordinados possam mostrar criatividade, bem como, criar novas formas de resolver os problemas gerados pelo excesso de burocracia e fazer escolhas, visando identificar oportunidades, bem como otimizar procedimentos visando aumentar sua produtividade (SÓREA DAC, et al., 2006). Ser resiliente é ser capaz de ultrapassar os limites interiores, sendo para isto, motivado a desenvolver habilidades pessoais, as quais possibilitam a promoção de sua autorrealização (BRANDÃO JM, et al., 2011).

\section{CONSIDERAÇÕES FINAIS}

Diante dos objetivos traçados no presente estudo, a literatura pertinente à resiliência dos enfermeiros revela pontos reflexivos. As exigências dos tempos modernos têm favorecido a impessoalidade das relações, a exiguidade de tempo, fazendo com que os enfermeiros se esqueçam de que não são máquinas, e que possuam o direito ao erro, mesmo que o ideal preconizado seja a perfeição, o que os tornam mais susceptíveis 
ao desequilíbrio. Contudo, com a aplicação dos conceitos do pensamento lateral e da resiliência na execução das atividades dos enfermeiros a estimulação da imaginação criativa possibilitam a ultrapassar diversas barreiras. Apesar da sobrecarga de atividades os enfermeiros conseguem utilizar seus talentos criativos e encontram novas soluções para os problemas, para contribuir com o crescimento e o desenvolvimento das instituições de saúde.

\section{REFERÊNCIAS}

1. ANDRADE BRV, et al. A resiliência do enfermeiro no cuidado à criança que vivencia a terminalidade. Rev. Enferm. UFSM, Santa Maria, v10, p. 1-18, 2020.

2. BIANCHINI DSC, DELL'AGLIO DD. Processo de resiliência no contexto de hospitalização: um estudo de caso. Paidéia, 2007; 35 (16) 427-436.

3. BONO E. O momento atual pela inovação. Entrevista. HSM Management, 2003; 37 (7) 44-49.

4. BRANDÃO JM, et al. A construção do conceito de resiliência em psicologia: discutindo as origens. Paidéia, $2011 ; 21$ (49) 263-271.

5. BRANT LC, DIAS EC. Trabalho e sofrimento em gestores. Caderno de Saúde Pública, 2004; 20(4) 942-949.

6. BRASIL. Política Nacional de Promoção da Saúde. Ministério da Saúde, 2010; (7) 60p.

7. CECHIN P, et al. Alterações vivenciadas por profissionais de enfermagem que apresentam dupla jornada de trabalho. Rev enferm UFPE on line, 2014; 8(11) 3855-3861.

8. CRUZ EJER, et al. Resiliência como objeto de estudo da saúde do trabalhador: uma revisão narrativa. J. res.: fundam. care. online 2018; 10(1) 283-288.

9. FERREIRA C, et al. Avaliação de esperança e resiliência em pessoas em tratamento hemodialítico. Rev Enferm UFSM, 2018; 8(4) 702-716.

10. GOLDSTEIN TS. Entre o conceito e a metáfora: a resiliência como abordagem do humano a partir da física dos materiais. O Mundo da Saúde, 2012; 36 (2) 327-33.

11. LIMA GS, et al. Resiliência, qualidade de vida e sintomas depressivos entre idosos em tratamento ambulatorial. Rev. Latino-Am. Enfermagem, 2019; 27, e3212.

12. MACEDO ABT, et al. Estresse psicossocial e resiliência: um estudo em profissionais da enfermagem. Rev. Enferm. UFSM, 2020; (10), e25, 1-17.

13. MENDES JM. Risco, Vulnerabilidade Social e Resiliência: Conceitos E Desafios. R. gest. sust. ambient., 2018; 7, n. esp. 463-492.

14. MONTEIRO MP, MARX LC. Pensamento lateral e resiliência como facilitadores das ações da gestão de enfermagem. Revistas Gerenciais, 2006; 5(2) 45-53.

15. PEIXOTO ALS. Considerações gerais sobre a alienação mental (1837). Rev. latinoam. psicopatol. Fundam, $2013 ; 16$ (4) 642-682.

16. REIS LA, MENEZES TMO. Religiosidade e espiritualidade nas estratégias de resiliência do idoso longevo no cotidiano. Rev. Bras. Enferm, 2017; 70 (4) 761-766.

17. SILVA JLL, MELO ECP. Estresse e implicações para o trabalhador de enfermagem. Informe-se em promoção da saúde, 2006; 2 (2) 16-18.

18. SILVA MJM, et al. Atividades gerenciais desempenhadas pelo enfermeiro no centro cirúrgico: obstáculos enfrentados pelo profissional no setor. Revista Eletrônica Acervo Saúde, 2019; (17), e652.

19. SÓREA DAC, et al. A resiliência como objeto de investigação na enfermagem e em outras áreas: uma revisão. Escola Anna Nery de Enfermagem, 2006; 10(3) 547-551.

20. SOUZA MTS, CERVENY CMO. Resiliência psicológica: revisão da literatura e análise da produção cientifica. Revista Interamericana de Psicologia, 2005; 40 (1)119-126. 